\title{
On Frequency Distributions of Wind Speed and Direction in Turbulent Flow*
}

\author{
By S. Syōno and H. Tanaka \\ Geophysical Institute, Tokyo University, Tokyo \\ (Manuscript received 2 November 1965, in revised form 15 March 1966)
}

\begin{abstract}
By applying the theory of random walk to a turbulent flow, the frequency distribution functions of wind speed and wind direction are derived. The turbulence in the flow is assumed to be homogeneous and isotropic in the horizontal, and further the phase angles of each component of the Fourier series of the finite variable wind speed are assumed to be at random and independent of each other.

Further by using the frequency distribution functions of wind speed, the diffusivity in the horizontal is obtained. Because there is no conclusive Lagrangian correlation function, we discussed the diffusivity using some correlation functions.

The theory is tested by using the records obtained in the Research Institute of Atomic Energy in Tokai Mura, Ibaraki Prefecture.
\end{abstract}

\section{Introduction}

It is usually considered that the fluctuation of wind speed in a turbulent flow obeys the Gaussian law. Hesselberg-Björkdal (1929) derived the law in an analogous manner with the kinetic theory of gases. But we cannot use this model, partly because the concept of scale of turbulence is not included in this model. Ertel (1929) obtained a frequency distribution function of wind direction using the result of Hesselberg-Björkdal, in which the maximum turbulent wind speed was treated as infinite. Different from molecules in a gas, the speed of a parcel in a fluid cannot be infinitely large, because of the finite kinetic energy and because of the basic assumption of continuity in the regime of hydrodynamics.

The authors have attempted to derive the frequency distribution functions of wind speed using the theory of random walk. The method is essentially the same with that of the previous paper of the senior author in 1953.

Now let us consider two sufficiently but finitely long records of wind speed from a very long record of wind speed, where the turbulence is stationary. Generally, the curves

* Division of Meteorology, Contribution No. 146 of the records are different from one another, in spite of the energy spectra are the same. The curves of finite length can be expressed in a Fourier series. In order to get the energy spectrum, phase angles are eliminated from the calculation. When we obtain the correlation functions, we assume that phase angles do not depend on the difference of two spatial or time distance in the domain but depend on the initial state or the non-linear coupling. On the contrary an instantaneous wind speed depends on both the amplitudes and the phase angles. Our main concern is the phase angles.

According to Priestley (1959), the frequency range of isotropy in the atmosphere is expressed as $N z / U_{0} \geq 0.6$, where $N$ is the frequency, $z$ the height, $U_{0}$ the mean speed of the wind. When $z=65 \mathrm{~m}, U_{0}=8.4 \mathrm{~m} / \mathrm{sec}$, the lower frequency limit of isotropy is $0.84 / \mathrm{sec}$. This value of $N$ is too large compared with the frequencies which are predominant in the microscale motion. In this case the turbulent field should be naturally considered as anisotropic. However, first we assume the turbulence is isotropic in the horizontal, and later treat an anisotropic turbulence.

In the first part, the frequency distribution function of wind speed is obtained. In the second part, the frequency distribution func- 
tion of wind direction are also obtained theoretically using the above function of wind speed.

In our theoretical treatment, we assumed that the maximum value of wind speed has a finite upper limit. Here the maximum speed is the sum of amplitudes of all components in a Fourier series of the curve of a record of wind speed.

\section{A model of turbulent flow}

In a fluid, physical elements of parcels are changing continuously in space. In the kinetic theory of gases, molecules are discrete and there are no interactions between them. On the other hand, in a continuous fluid, there are interactions between the elements. From this reasoning it is not allowed for elements to move arbitrarily, then analogy of the kinetic theory of gases is not adequate. We must treat a fluid as a continuum. Amplitudes and phase angles are two variables in a Fourier series. It is not easy to obtain the Gaussian law under any assumption of amplitudes provided that one eliminates phase angles. On the other hand it is easy to get the law under an assumption of randomness of phase angles without any restriction of amplitudes.

The time velocity curve of the record of wind speed is expressed by a Fourier series

$$
\boldsymbol{u}_{n}=\boldsymbol{U}_{0}+\sum_{i=1}^{n} \boldsymbol{C}_{i} \cos \left(\frac{2 \pi i}{T} t-\boldsymbol{1} \cdot \boldsymbol{\varphi}_{i}\right)
$$

where

$$
\boldsymbol{U}_{0}=\frac{1}{T} \int_{0}^{T} \boldsymbol{u}_{n}(\lambda) d \lambda
$$

$\boldsymbol{U}_{0}$ is the mean velocity, $\boldsymbol{C}_{i}$ the vector amplitude of each elementary component, $\varphi_{i}$ the phase angle, $\boldsymbol{I}$ the unit vector and $T$ the period of observation. Even if turbulent flow has the same mean speed and energy spectrum, the time-velocity curve does not coincide with each other (see Fig. 1). We assumed that phase angles of corresponding components of the two curves are not the same. In the problems concerning energy spectra and correlation function, the effect of phase angles disappears as shown in the following calculations,

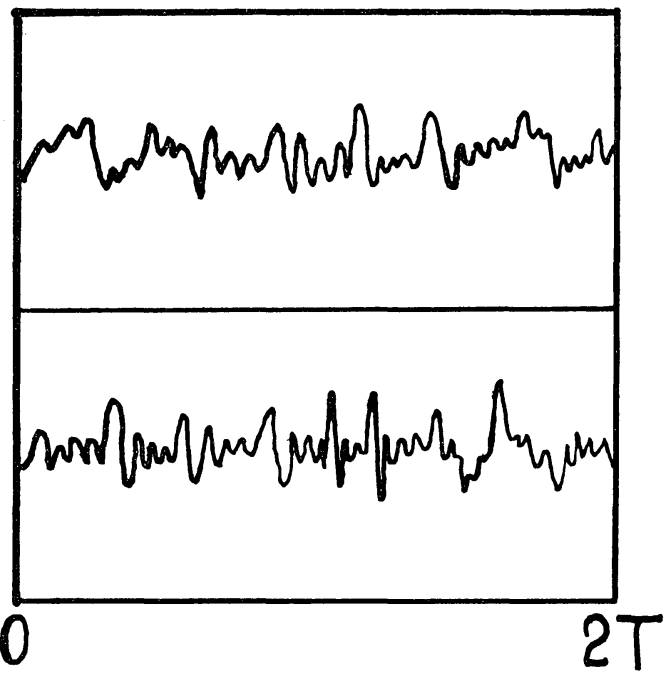

Fig. 1 Time-velocity curves of turbulence.

Kinetic energy :

$$
\begin{aligned}
K & =\frac{1}{2} \bar{u}_{n}^{2}=\frac{1}{2 T} \int_{0}^{T}\left\{U_{0}+\sum_{i} C_{i} \cos \left(\frac{2 \pi i}{T} t-\varphi_{i}\right)\right\}^{2} d t \\
& =\frac{1}{2}\left\{U_{0}{ }^{2}+\sum_{i} C_{i}^{2} \frac{1}{T} \int_{0}^{T} \cos ^{2}\left(\frac{2 \pi i}{T} t-\varphi_{i}\right) d t\right\}^{2} \\
& =K_{0}+\sum_{i} \frac{C_{i}^{2}}{2},
\end{aligned}
$$

Spatial correlation :

$$
\begin{aligned}
R_{x}= & \frac{1}{\bar{u}_{n}^{2}} \cdot \frac{1}{T} \int_{0}^{T}\left\{U_{0}+\sum_{i} C_{i} \cos \left(\frac{2 \pi i}{T} t-\varphi_{i}\right)\right\} \\
& \times\left\{U_{0}+\sum_{i} C_{i} \cos \left(\frac{2 \pi i}{T} t-\varphi_{i}+\frac{2 \pi i}{T U_{0}} x\right)\right\} d t \\
& =\frac{1}{\bar{u}_{n}^{2}} \cdot \frac{1}{T} \sum_{i} C_{i}{ }^{2} \int_{0}^{T} \cos ^{2}\left(\frac{2 \pi i}{T} t-\varphi_{i}\right) \cos \frac{2 \pi i}{U_{0}} x d t \\
& =\sum_{i} \frac{C_{i}{ }^{2}}{2} \cos \frac{2 \pi i}{U_{0}} x,
\end{aligned}
$$

Spectral function: $\quad F(i)=\frac{C_{i}{ }^{2}}{2}$.

The above assumption introduces no difficulty in the current theory of turbulence. Phase angles are the undiscussed freedom. In our investigation by which the distribution functions are derived, phase angles are primarily important. We can express the velocity at any instant in statistical sense as

$$
\boldsymbol{u}_{n}=\boldsymbol{U}_{0}+\sum_{i=1}^{N} \boldsymbol{C}_{i} \cos \left(\boldsymbol{1} \cdot \boldsymbol{\varphi}_{i}\right)
$$

and phase angles are at random and inde- 
pendent of each other rank. In other words, phase angles may take any values according to the initial conditions. Further non-linear interaction in turbulent field changes phase angles very complicatedly, so that the assumption that the phase angles are at random and independent may not be unreasonable.

\section{The frequency distribution of wind speed}

Now we consider the probability that the velocity fluctuation $\boldsymbol{u}_{n}{ }^{\prime}\left(=\boldsymbol{u}_{n}-\boldsymbol{U}_{0}\right)$ lies in the range $\left|\boldsymbol{u}_{n}^{\prime}\right|<\mid U$, where $U$ is an arbitrary value of wind speed. The probability can be written as follows:

$$
\begin{aligned}
& P_{n}(U) d U=d U\left(\frac{1}{2 \pi}\right)^{n-1} \overbrace{\int_{-\pi}^{\pi} \ldots . .}^{n-2} \int_{-\pi}^{\pi} \\
& \times \int d \varphi_{n-1} d \varphi_{n-2} \cdots d \varphi_{1} .
\end{aligned}
$$

In the above integration, $\varphi_{1}, \cdots, \varphi_{n-2}$ take all values between $-\pi$ and $\pi$, while $\varphi_{n-1}$ takes only such values as $\left|\boldsymbol{u}_{n}{ }^{\prime}\right|<U$ for each set of of $\varphi_{1}, \cdots, \varphi_{n-2}$. When the following integral

$$
U \int_{0}^{\infty} J_{1}(U t) J_{0}\left(S_{n} t\right) d t= \begin{cases}1 & S_{n}<U \\ 0 & S_{n}>U\end{cases}
$$

is introduced, the range of $\varphi_{n-1}$ may be taken the values between $-\pi$ and $\pi$, where $t$ denotes the integral variable. Inserting (7) into (6), then we get

$$
\begin{aligned}
& P_{n}(U) d U=d U\left(\frac{1}{2 \pi}\right)^{n-1} \overbrace{\int_{-\pi}^{\pi} \ldots \cdots \int_{-\pi}^{n-1}}^{\pi} \\
& \times \int_{0}^{\infty} U J_{1}(U t) J_{0}\left(S_{n} t\right) d t d \varphi_{n-1} \cdots d \varphi_{1},
\end{aligned}
$$

where

$$
S_{n}=\left(S_{n-1}{ }^{2}-2 S_{n-1} C_{n} \cos \varphi_{n-1}+C_{n}^{2}\right)^{1 / 2}
$$

and then we can use the formula of Bessel function :

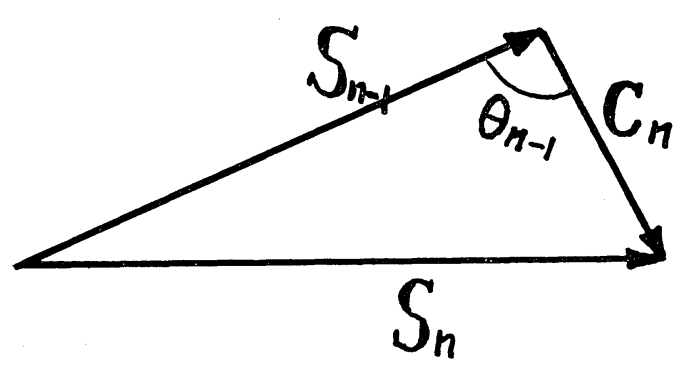

Fig. 2 .

$$
\begin{aligned}
J_{0}\left(S_{n} t\right) & =J_{0}\left(t \sqrt{S_{n-1}^{2}-2 S_{n-1} C_{n} \cos \varphi_{n-1}+C_{n}^{2}}\right) \\
& =J_{0}\left(S_{n-1} t\right) J_{0}\left(C_{n} t\right) \\
& +2 \sum_{\nu=1}^{\infty} J_{\nu}\left(S_{n-1} t\right) J_{r}\left(C_{n} t\right) \cos \nu \varphi_{n-1} .
\end{aligned}
$$

Putting (9) into (8), the second term of right hand side vanishes and we get

$$
\begin{aligned}
& P_{n}(U) d U=d U\left(\frac{1}{2 \pi}\right)^{n-2} \overbrace{\int_{-\pi}^{\pi} \cdots \cdots}^{n-1} \ldots \int_{-\pi}^{\pi} \int_{0}^{\infty} \\
& \quad \times U J_{1}(U t) J_{0}\left(S_{n-1} t\right) J_{0}\left(C_{n} t\right) d t d \varphi_{n-2} \cdots d \varphi_{1} .
\end{aligned}
$$

Similarly repeating integration with respect to $\varphi$, we get finally

$$
P_{n}(U) d U= \begin{cases}U d U \int_{0}^{\infty} J_{1}(U t) \prod_{i=1}^{n} J_{0}\left(C_{i} t\right) d t & U \leq U_{m} \\ 0 & U>U_{m} .\end{cases}
$$

$U_{m}$ means the sum of amplitudes of elementary components from rank 1 to rank $n$, i.e., $U_{m}=\sum_{i=1}^{n} C_{i}$. $S_{n}$ denotes absolute value of vector sum of amplitudes from rank 1 to rank $n$. For $U>U_{m}$, value of probability vanishes. Using asymptotic expansion of Bessel function for a small value of $t$, we get

$J_{0}\left(C_{\imath} t\right) \simeq \exp \left[-\frac{\sigma^{2}}{2} t^{2}\right]\left\{1-\frac{(\sigma t)^{4}}{16}-\mathrm{O}(\sigma t)^{6}\right\}$,

where

$$
\sigma^{2}=\sum_{i=1}^{n} \frac{C_{i}{ }^{2}}{2}, \quad \sigma^{4}=\sum_{i=1}^{n} C_{i}{ }^{4} .
$$

Taking only the first term of the asymptotic expansion, (10) becomes approximately

$P_{n}(U) d U \simeq d U \int_{0}^{\infty} U J_{1}(U t) \exp \left[-\frac{\sigma^{2}}{2} t^{2}\right] d t$

$=d U \int_{0}^{\infty} J_{1}(x) \exp \left[-\frac{\sigma^{2}}{2 U^{2}} x^{2}\right] d x$

$=\left\{\begin{array}{r}\frac{U^{2} d U}{2 \sigma^{2}} \exp \left[-\frac{U^{2}}{2 \sigma^{2}}\right]_{1} F_{1}\left(1 ; 2 ; \frac{U^{2}}{2 \sigma^{2}}\right) U<U_{m} \\ 0 \quad U>U_{m},\end{array}\right.$

where ${ }_{1} F_{1}$ shows the confluent hypergeometric function and $x=U t$.

In order to obtain the frequency distribution of wind speed, that is, the probability which $\left|\boldsymbol{u}_{n}^{\prime}\right|$ lines in the range $U \sim U+d U$, we 
use the following relation :

$$
2 \pi U F_{n}(U) d U=\frac{d P_{n}(U)}{d U} d U
$$

$$
\begin{aligned}
2 \pi U F_{n}(U) d U & =d U\left\{\frac{d}{d U} \int_{0}^{\infty} U J_{1}(U t) \prod_{i=1}^{n} J_{0}\left(C_{i} t\right) d t\right\}=d U \int_{0}^{\infty} U t J_{0}(U t) \prod_{i=1}^{n} J_{0}\left(C_{i} t\right) d t \\
& \simeq d U \int^{\infty} U t J_{0}(U t) \exp \left[-\frac{\sigma^{2}}{2} t^{2}\right] d t=\frac{d U}{U} \int_{0}^{\infty} x J_{0}(x) \exp \left[\frac{\sigma^{2}}{2 U^{2}} x^{2}\right] d x \\
& = \begin{cases}\frac{U d U}{\sigma^{2}} \exp \left[-\frac{U^{2}}{2 \sigma^{2}}\right] & U<U_{m} \\
0 & U>U_{m} .\end{cases}
\end{aligned}
$$

4. The second approximation of the frequency distribution of wind speed

The above result is obtained by taking the first term of the asymptotic expansion of the Bessel function for small value of $t$. In order to get more exact integrated value,
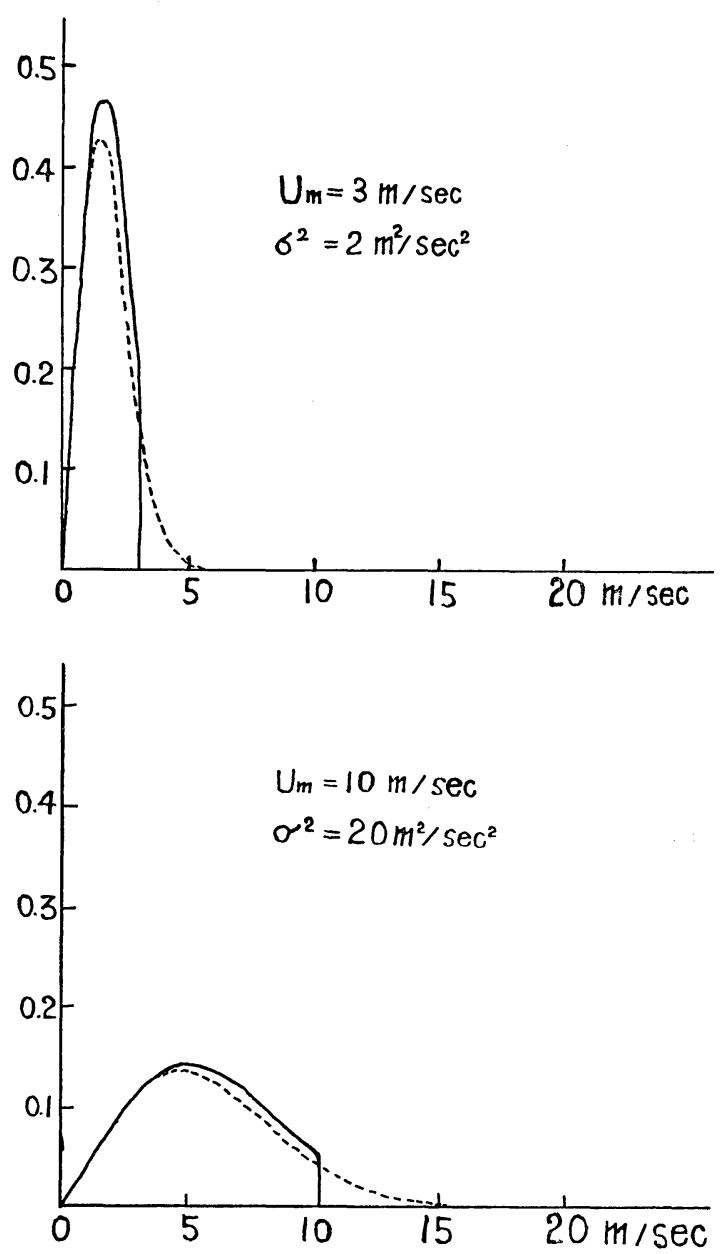

we must go back to the integral in (15). For large value of $t$, the asymptotic expansion does not express exactly the Bessel function. By the reason that the probability (15) between $0<U<U_{m}$ does not equal unity, but exactly speaking it should be equal to unity. Using
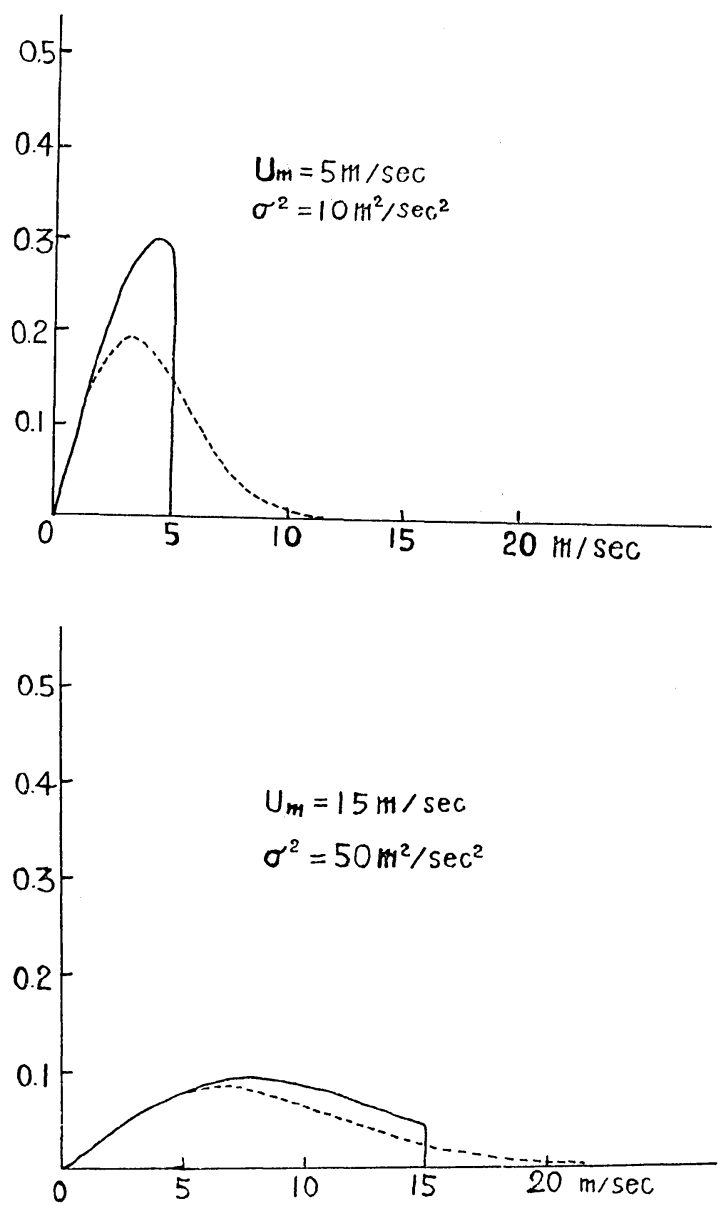

Fig. 3. Curves of (16) for various values of $U_{m}$ and $\sigma^{2}$. Dashed lines are case of $\alpha=0$. 
the latter condition, we shall obtain an approximate expression. In the range $0<U<U_{m}$, the second approximation is assumed as follows:

$$
2 \pi U \hat{F}_{n}(U) d U=\frac{U}{\sigma^{2}} \exp \left[-\begin{array}{c}
U^{2} \\
2 \sigma^{2}
\end{array}\right]\left(1+\alpha U^{2}\right) d U,
$$

where $\hat{F}_{n}(U)$ is the modified form of $F_{n}(U)$, $\alpha$ the constant which is determined by the following condition:

$$
2 \pi \int_{0}^{U_{m}} U \hat{F}_{n}(U) d U=1 .
$$

Inserting (16) into (17), we get

$$
\begin{gathered}
\alpha=\left(\sigma^{2}-\int_{0}^{U_{m}} U \exp \left[-\frac{U^{2}}{2 \sigma^{2}}\right] d U\right) / \\
\left(\int_{0}^{U_{m}} U^{3} \exp \frac{U^{2}}{2 \sigma^{2}} d U\right) .
\end{gathered}
$$

Here

$$
\begin{aligned}
\int_{0}^{U_{m}} U \exp \left[-\frac{U^{2}}{2 \sigma^{2}}\right] d U & =\sigma^{2}\left(1-\exp \left[-\frac{U_{m}^{2}}{2 \sigma^{2}}\right]\right) \\
\int_{0}^{U_{m}} U^{3} \exp \left[-\frac{U^{2}}{2 \sigma^{2}}\right] d U & =2 \sigma^{4}\left(1-\exp \left[-\frac{U_{m}^{2}}{2 \sigma^{2}}\right]\right) \\
& -\sigma^{2} U_{m}^{2} \exp \left[-\frac{U_{m}^{2}}{2 \sigma^{2}}\right],
\end{aligned}
$$

\begin{tabular}{|c|c|c|}
\hline$\sigma^{2}\left(\mathrm{~m}^{2} / \mathrm{sec}^{2}\right) \quad U_{m}(\mathrm{~m} / \mathrm{sec})$ & 25 & 4 \\
\hline 15 & $2.0 \times 10^{-5}$ & - \\
\hline 5 & $0.0 \times 10^{-5}$ & 4. $25 \times 10^{-2}$ \\
\hline
\end{tabular}

then $\alpha$ becomes as follows :

$$
\alpha=1 /\left\{2 \sigma^{2}\left(\exp \left[\frac{U_{m}^{2}}{2 \sigma^{2}}\right]-1\right)-U_{m}^{2}\right\} .
$$

Table 1. Value of $\alpha$.

Fig. 3 shows the curves of (16).

\section{The frequency distribution of wind direc- tion}

Here we shall obtain the frequency distribution of wind direction. The mean flow speed is assumed to be $U_{0}$. In this case (see Fig. 4), the following relations are satisfied among $V, U, U_{0}, \psi$ and $\theta$ :

$$
\left\{\begin{array}{l}
U^{2}=V^{2}+U_{0}^{2}-2 V U_{0} \cos \phi \\
V \sin \psi=U \sin \theta
\end{array}\right.
$$

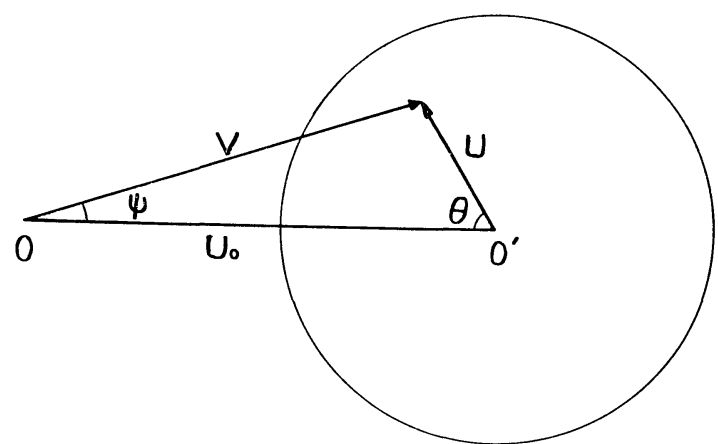

Fig. 4. Figure of relation among $V, U, U_{0}$, $\psi$ and $\theta$.

Using these relations (19), we get the following transformation of variables:

$$
d U d \theta=\left|\begin{array}{ll}
\frac{\partial U}{\partial V} & \frac{\partial U}{\partial \phi} \\
\frac{\partial \theta}{\partial V} & \frac{\partial \theta}{\partial \phi}
\end{array}\right| d V d \psi=\frac{V}{U} d V d \psi
$$

Then

$$
U d U d \theta=V d V d \theta .
$$

By this transformation of variables, we get

$$
\hat{F}_{n}(U) U d U d \theta=\hat{F}_{n}(V, \phi) V d V d \phi,
$$

where

$$
\begin{aligned}
& \hat{F}_{n}(V, \phi)=\frac{1}{2 \pi \sigma^{2}} \exp \left[-\frac{V^{2}+U_{0}^{2}-2 V U_{0} \cos \phi}{2 \sigma^{2}}\right] \\
& \quad \times\left\{1+\alpha\left(V^{2}+U_{0}^{2}-2 V U_{0} \cos \phi\right)\right\} .
\end{aligned}
$$

Integrating this probability function (21) with respect to $V$, we can obtain the frequency distribution function of wind direction. In this integration, we must consider two cases.

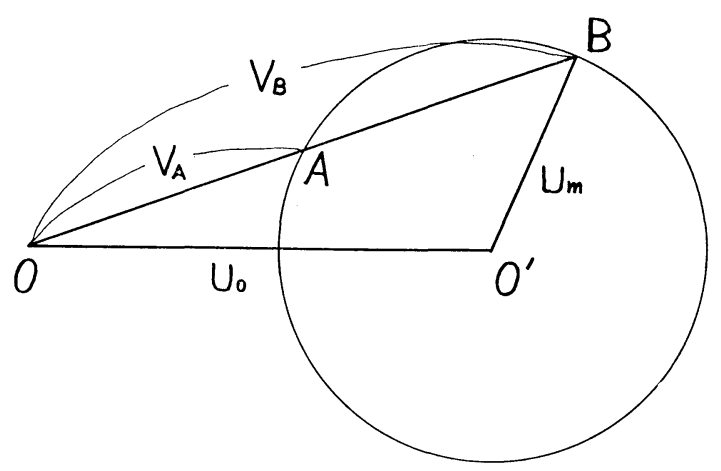

Fig. 5. Figure of relation among $U_{0}, U_{m}, V_{A}$ and $V_{B}$ in the case $U_{0} \geq U_{m}$. 


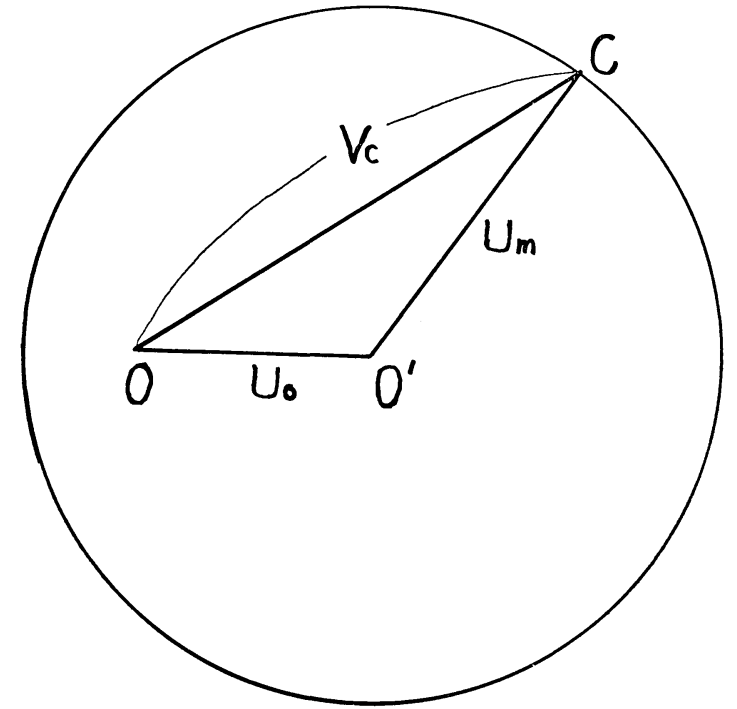

Fig. 6. Figure of relation among $U_{0}, U_{m}$ and $V_{c}$ in the case $U_{0}<U_{m}$.
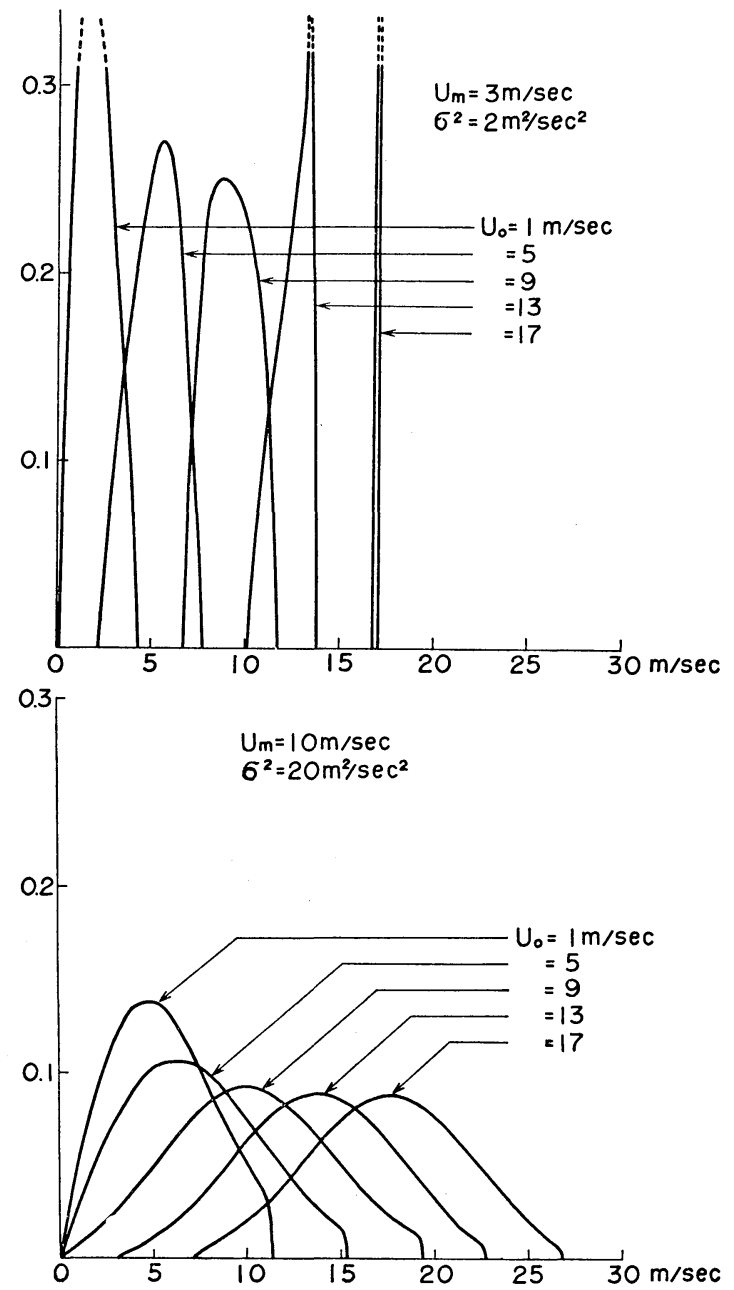

One is $U_{0}>U_{m}$, another is $U_{0}<U_{m}$. For the case $U_{0}>U_{m}$ (Fig. 5), we must integrate $\hat{F}_{n}(V, \phi) V d V d \phi$ with respect to $V$ from $V_{A}$ $\left(=U_{0} \cos \phi-\sqrt{\left.U_{m}^{2}-U_{0}^{2} \sin ^{2} \phi\right)}\right.$ to $V_{B}\left(=U_{0} \cos \psi\right.$ $\left.+\sqrt{U_{m}{ }^{2}-U_{0}{ }^{2} \sin ^{2} \phi}\right)$. On the other hand, for the case $U_{0}<U_{m}$ (Fig. 6), we must integrate $\hat{F}_{n}(V, \psi) V d V d \psi$ from 0 to $V_{c}\left(=U_{0} \cos \psi\right.$ $+\sqrt{U_{m}^{2}-U_{0}^{2} \sin ^{2} \phi}$.

Now let us integrate $\hat{F}_{n}(V, \phi) V d V d \psi$ with respect to $V$ and we obtain the following results :

$$
\begin{aligned}
& \text { (i) } U_{0}>U_{m} \\
& \hat{F}_{n}(\psi) d \psi=\frac{U_{0} \cos \psi}{\sqrt{2 \pi} \sigma} \exp \left[-\frac{U_{0}^{2} \sin ^{2} \psi}{2 \sigma^{2}}\right] \\
& \times\left(1+\alpha U_{0}^{2} \sin ^{2} \varphi+2 \alpha \sigma^{2}\right) \\
& \times \Phi\left(\sqrt{\frac{U_{m}^{2}-U_{0}^{2} \sin ^{2} \psi}{2 \sigma^{2}}}\right) d \psi-\frac{\alpha U_{0} \cos \psi}{\pi}
\end{aligned}
$$
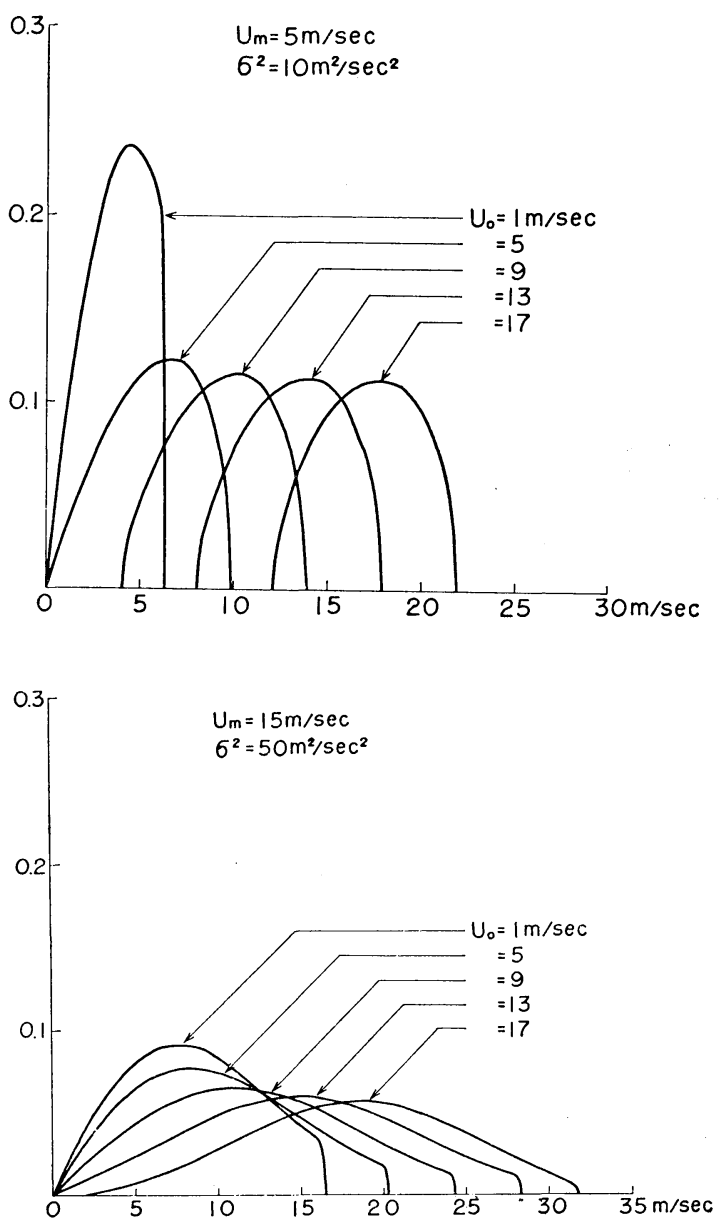

Fig. 7. Distribution curves of wind speed for various values of $U_{0}, U_{m}$ and $\sigma^{2}$. 

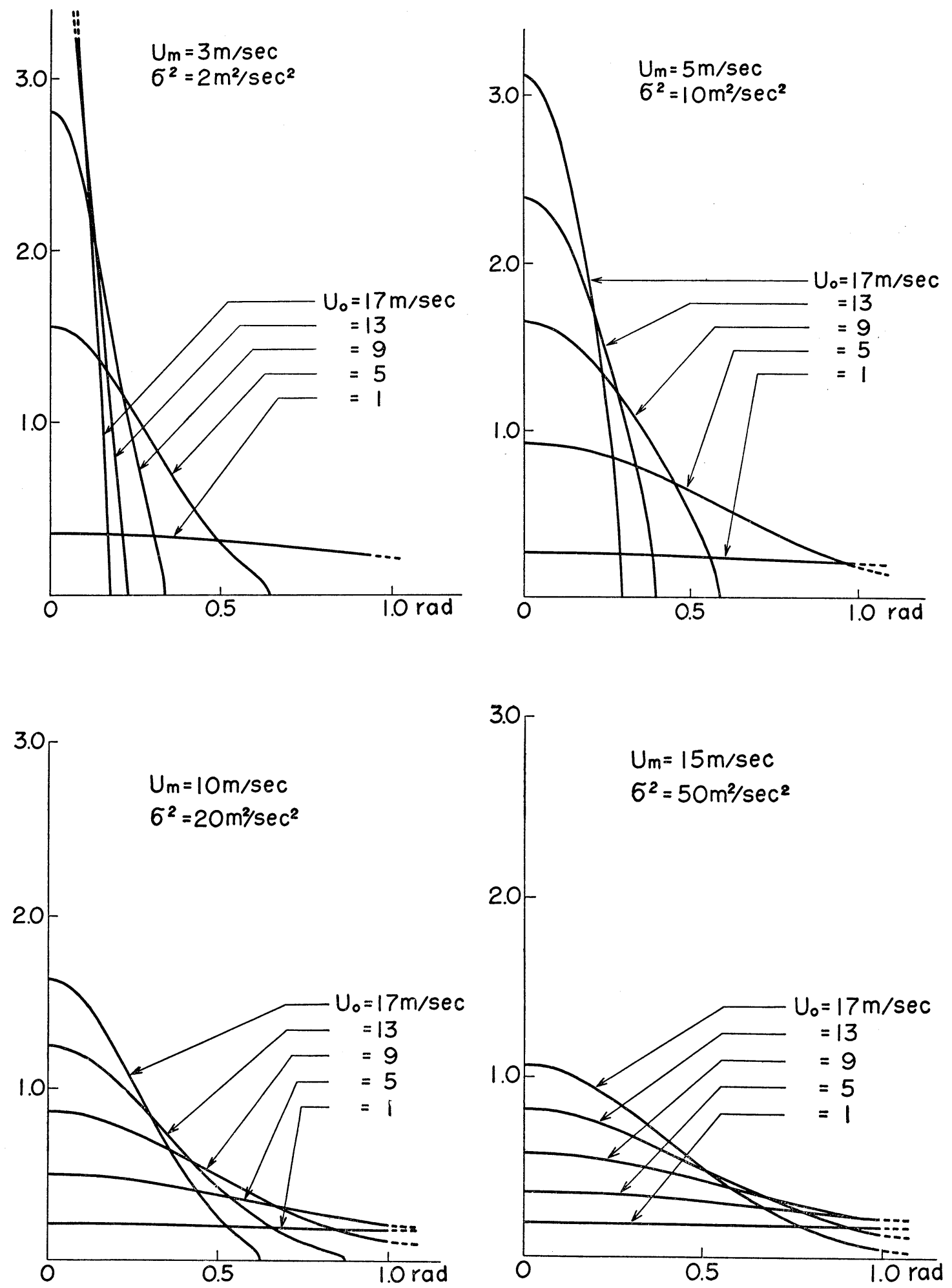

Fig. 8. Distribution curves of wind direction for various values of $U_{0}, U_{m}$ and $\sigma^{2}$. 


$$
\begin{aligned}
& \times \sqrt{U_{m}^{2}-\bar{U}_{0}^{2} \sin ^{2} \psi} \exp \left[-\frac{U_{m}^{2}}{2 \sigma^{2}}\right] d \phi, \\
& \quad\left(|\phi|>\sin ^{-1} \frac{U_{m}}{U_{0}}\right) .
\end{aligned}
$$

(ii) $U_{0}<U_{m}$

$\hat{F}_{n}(\phi) d \psi=\frac{U_{0} \cos \phi}{2 \sqrt{2 \pi} \sigma} \exp \left[-\frac{U_{0}^{2} \sin ^{2} \psi}{2 \sigma^{2}}\right]$

$\times\left(1+\alpha \sigma^{2}+\alpha U_{0}^{2} \sin ^{2} \psi\right)$

$\times\left[\Phi\left(\sqrt{\frac{U_{m}{ }^{2}-U_{0}^{2} \sin ^{2} \psi}{2 \sigma^{2}}}\right)+\Phi\left(\frac{U_{0} \cos \phi}{\sqrt{2} \sigma}\right)\right] d \psi$

$+\frac{1}{2 \pi}\left(1+2 \alpha \sigma^{2}+\alpha U_{0}^{2} \sin ^{2} \psi\right) \exp \left[-\frac{U_{0}^{2}}{2 \sigma^{2}}\right] d \psi$

$-\frac{1}{2 \pi}\left(1+2 \alpha \sigma^{2}+\alpha U_{m}^{2}+\alpha U_{0} \cos \phi\right.$

$\left.\times \sqrt{U_{m}^{2}-U_{0}^{2} \sin ^{2} \phi}\right) \exp \left[-\frac{U_{m}^{2}}{2 \sigma^{2}}\right] d \phi, \quad(|\phi| \leqq \pi)$.

Here $\Phi(x)$ denotes the following function:

$$
\Phi(x)=\frac{2}{\sqrt{ }} \frac{-}{\pi} \int_{0}^{x} \exp \left(-t^{2}\right) d t
$$

Fig. 7 shows the curves of values of $\hat{F}_{n}(\phi)$ given by (22) and (23) for various values of $U_{0}, U_{m}$ and $\sigma^{2}$.

\section{The frequency distribution functions of total wind speed}

In the same way, integrating $\hat{F}_{n}(V, \psi) V d V d \psi$ with respect to $\phi$ from $-\theta_{1}$ to $\theta_{1}$ $\left(=\cos ^{-1}\left(\left(V^{2}+U_{0}{ }^{2}-U_{m}{ }^{2}\right) / 2 V U_{0}\right)\right.$ for the case $U_{0}>U_{m}$, and from $-\pi$ to $\pi$ for the case $U_{0}<U_{m}$.

(i) $U_{0}>U_{m}$

$$
\begin{aligned}
& \hat{F}_{n}(V) V d V=\frac{1}{2 \sqrt{\pi} \sigma^{2}} \exp \left[-\frac{\left(V-U_{0}\right)^{2}}{2 \sigma^{2}}\right] \\
& \times\left\{1+\alpha\left(V-U_{0}\right)^{2}+\alpha \frac{\partial}{\partial\left(\frac{U_{0} V}{\sigma^{2}}\right)}\right\} \\
& \times \Psi\left(\frac{2 U_{0} V}{\sigma^{2}}, \cos ^{-1} \frac{V^{2}+U_{0}-U_{m}^{2}}{2 U_{0} V}\right) V d V, \\
& \quad\left(U_{0}-U_{m}<V<U_{0}+U_{m}\right) .
\end{aligned}
$$

(ii) $U_{0}<U_{m}$

$$
\begin{aligned}
& \hat{F}_{n}(V) V d V=\frac{1}{2 \sqrt{\pi} \sigma^{2}} \exp \left[-\frac{\left(V-U_{0}\right)^{2}}{2 \sigma^{2}}\right] \\
& \quad \times\left\{1+\alpha\left(V-U_{0}\right)^{2}+\alpha \frac{\partial}{\partial\left(\frac{U_{0} V}{\sigma^{2}}\right)}\right\}
\end{aligned}
$$

$$
\times \Psi\left(\frac{2 U_{0} V}{\sigma^{2}}, \pi\right) V d V, \quad\left(0<V<U_{0}+U_{m}\right) .
$$

Here $\Psi(\beta, \theta)$ denotes the following function:

$$
\Psi(\beta, \theta)=\frac{2}{\sqrt{\pi}} \int_{0}^{\theta} \exp \left[-\beta \sin ^{2} \frac{\varphi}{2}\right] d \varphi .
$$

Fig. 8 shows the curves of $\hat{F}_{n}(V) V$ obtained from (25) and (26) for various values of $U_{0}$, $U_{m}$ and $\sigma^{2}$.

\section{Discussions using the actual data}

It is important to test assumptions of the present theory by comparing with actual observations. In order to test this assumption, we used the data which were obtianed in September 15, 1958 on the meteorological tower (65 $\mathrm{m}$ high) in the Research Institute of Atomic Energy of Tokai Mura, Ibaraki Prefecture. The wind speed and the wind direction are recorded by an aerovane anemometer. The Research Institute of Atomic Energy is built near the coast of the Pacific Ocean in the north-eastern part of Kanto plane. The records are read at every 7.5 seconds from $12: 00$ to $12: 30$ and the 240 pairs of the values of the wind speed and the wind direction were obtained. In Fig. 9 points show velocities at every instant in polar coordinates, when the amplitudes are the wind speeds and the azimuths are the wind directions. In order to eliminate the slow fluctuations of main flow, we take six mean values of the speed and the direction in every five

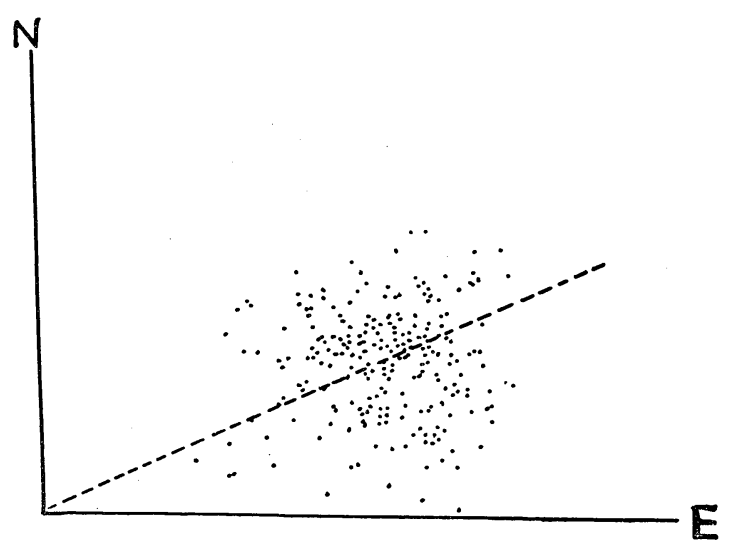

Fig. 9. Distribution of wind speed and direction at every 7.5 seconds from $12: 00$ to $12: 30$. 
minutes intervals (Fig. 10), then make them coincide to the mean values of thirty minutes (Fig. 11).
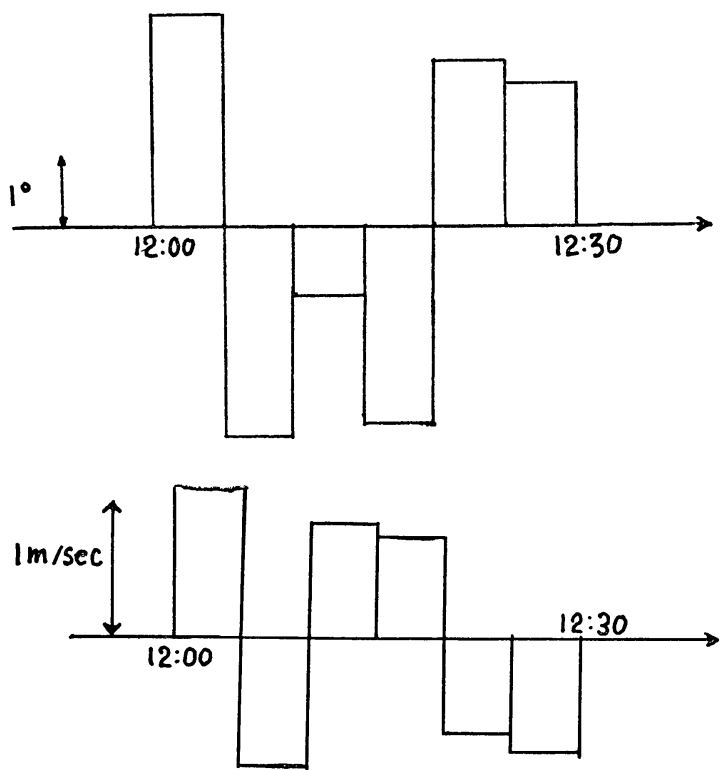

Fig. 10. Mean values of wind speed and wind direction at every 5 minutes.

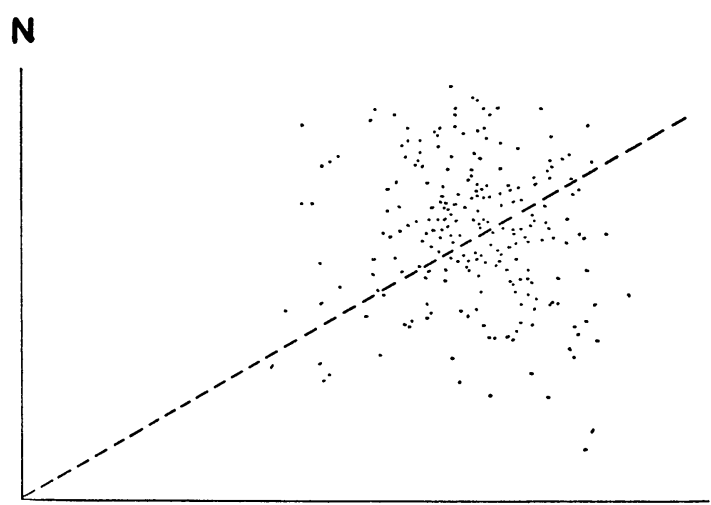

Fig. 11. Arranged distribution of wind speed and direction from Fig. 11 using mean values of Fig. 12.

Using the data above arranged, we calculated the various values as are written below:

$$
\begin{aligned}
& U_{0}=\frac{1}{240} \sum_{i} V_{i} \cos \phi_{i}, \\
& \left\{\begin{array}{l}
V_{x_{i}}=\left|V_{i} \cos \phi_{i}-U_{0}\right| \\
V_{y_{i}}=V_{i} \sin \phi_{i},
\end{array}\right.
\end{aligned}
$$

$$
\begin{gathered}
\rho=\left(\sum_{i} V_{x i} V_{y i}-\frac{\sum_{i} V_{x i} \sum_{i} V_{y i}}{240}\right) / \\
\left(\sum_{i} V_{x i}{ }^{2}-\frac{\left(\sum_{i} V_{x i}\right)^{2}}{240}\right)^{1 / 2}\left(\sum_{i} V_{y i}{ }^{2}-\frac{\left(\sum_{i} V_{y i}\right)^{2}}{240}\right)^{1 / 2}, \\
\left\{\begin{array}{l}
\sigma_{x}{ }^{2}=\frac{1}{239}\left(\sum_{i} V_{x i}{ }^{2}-\frac{\left(\sum_{i} V_{x i}\right)^{2}}{240}\right) \\
\sigma_{y}{ }^{2}=\frac{1}{239}\left(\sum_{i} V_{y i}{ }^{2}-\frac{\left(\sum_{i} V_{y i}\right)^{2}}{240}\right), \\
\sigma^{2}=\sigma_{x}{ }^{2}+\sigma_{y}{ }^{2},
\end{array}\right.
\end{gathered}
$$

where $U_{0}$ is the mean speed in the $x$-direction which is the mean direction of thirty minutes, $\rho$ the correlation coefficient between $x$ - and $y$-directions, $\sigma_{x}$ and $\sigma_{y}$ the standard deviations in the $x$-and $y$-directions respectively, $\sigma$ the standard deviation (Fig. 12). Carrying out the calculation we obtained the following values:

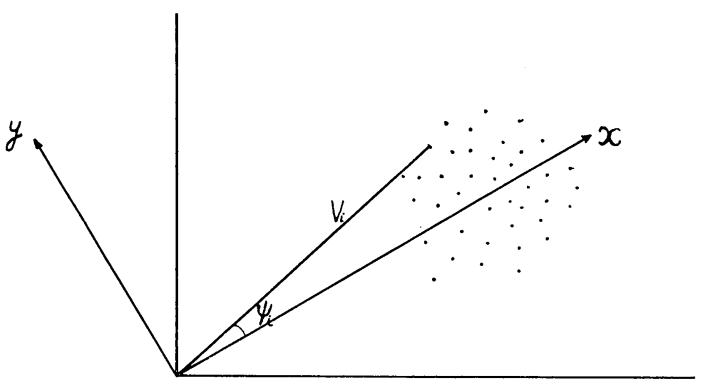

Fig. 12. Figure of $V_{i}$ and $\phi_{i}$.

$$
\left\{\begin{array} { l } 
{ U _ { 0 } = 8 . 4 1 \mathrm { m } / \mathrm { sec } } \\
{ \rho = - 0 . 0 4 9 , }
\end{array} \quad \left\{\begin{array}{l}
\sigma_{x}{ }^{2}=0.51 \\
\sigma_{y}{ }^{2}=0.78 \\
\sigma^{2}=1.28
\end{array}\right.\right.
$$

Further we carried out the Fourier analysis of the wind speed. Fourier components are represented

$$
\begin{aligned}
& \left\{\begin{array}{l}
A_{x}{ }^{(k)}=\frac{1}{120} \sum_{i} V_{x i} \cos \frac{2 \pi}{240} i k \\
B_{x}{ }^{(k)}=\frac{1}{120} \sum_{i} V_{x i} \sin \frac{2 \pi}{240} i k,
\end{array}\right. \\
& \left\{\begin{array}{l}
A_{y}{ }^{(k)}=\frac{1}{120} \sum_{i} V_{y i} \cos \frac{2 \pi}{240} i k \\
B_{y}{ }^{(k)}=\frac{1}{120} \sum V_{y i} \cos \frac{2 \pi}{240} i k .
\end{array}\right.
\end{aligned}
$$


Using these Fourier components, we obtained the maximum speed of the turbulence. It is shown

$$
U_{m}=\sum_{k=1}^{120}\left(A_{x}^{(k)^{2}}+B_{x}^{(k)^{2}}+A_{y}^{(k)^{2}}+B_{y}^{(k)^{2}}\right)^{1 / 2} .
$$

The standard deviations are also expressed using the Fourier components,

$$
\begin{aligned}
& \left\{\begin{array}{l}
\sigma_{x}^{2}=\frac{1}{2} \sum_{k=1}^{120}\left(A_{x}{ }^{(k)^{2}}+B_{x}^{(k)^{2}}\right) \\
\sigma_{y}^{2}=\frac{1}{2} \sum_{k=1}^{120}\left(A_{y}(k)^{2}+B_{y}{ }^{(k)^{2}}\right),
\end{array}\right. \\
& \sigma^{2}=\sigma_{x}^{2}+\sigma_{y}{ }^{2} .
\end{aligned}
$$

As the results of the calculation, we get

$$
\left\{\begin{array}{l}
U_{m}=16.37 \mathrm{~m} / \mathrm{sec} \\
\sigma_{x}{ }^{2}=0.51, \quad \sigma_{y}{ }^{2}=0.78 \\
\sigma^{2}=1.28 .
\end{array}\right.
$$

We can understand that values of the standard deviations which are obtained from (31) and (37) are the same.

(i) Discussions about randomness of phase angles

It seems that the value of $U_{m}$ is about $3 \mathrm{~m} / \mathrm{sec}$ from the rough estimation in Fig. 2 . But the value of $U_{m}$ which is got by the Fourier components is far larger than the observed value of $U_{m}$. This is because in the actual phenomena, the phase angles do not coincide with each other, on the other hand the calculated value of $U_{m}$ from the Fourier analysis is the value which is got in the case where all phase angles completely coincide with each other, and the probability of this case is vanishingly small. This discrepancy does not necessarily mean that phase angles are perfectly at random but indicates that there is large probability of randomness of phase angles.

(ii) Discussions about isotropy of turbulent flow in the Horizontal

The standard deviations and the correlation coefficient between two directions were obtained. Seeing these results, there is only a weak correlation between two directions but is fairly large difference between the standard deviations of two directions. Fig. 13 shows the probable ellipse which is represented by

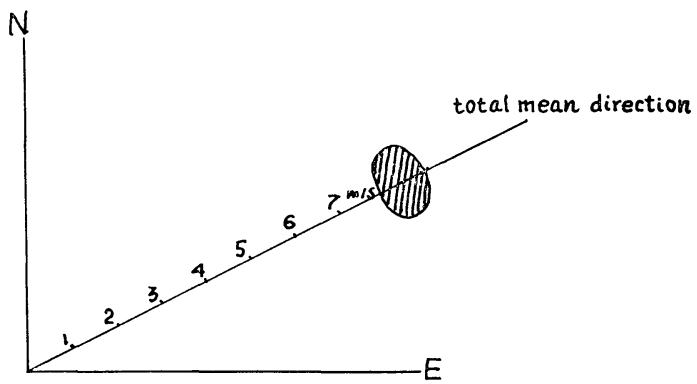

Fig. 13. Probable ellipse in which $500 \%$ of spots are contained.

$$
\frac{x^{2}}{\sigma_{x}^{2}}-\frac{2 \rho x y}{\sigma_{x} \sigma_{y}}+\frac{y^{2}}{\sigma_{y}^{2}}=\sqrt{1-\rho^{2}} \chi,
$$

where $\chi$ is the parameter of probability.

The fluctuation is more spread perpendicular to the main flow direction. In the case where turbulence is anisotropic, we have to slightly change the present theory. Fig. 14 shows the schematic figures in anisotropic turbulence corresponding to ones shown in Fig. 5 and Fig. 6. We find that the theoretical treatment is far more complex for this case. Further it may be important to examine whether wind direction and wind speed in turbulent flow obey the Markov's stochastic process or not.
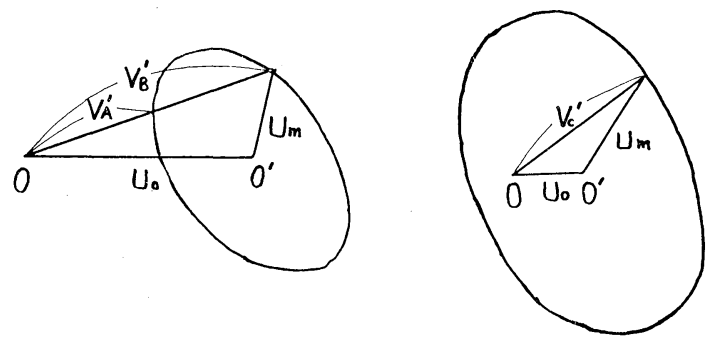

Fig. 14. Schematic figures in anistropic turbulence.

\section{(iii) Frequency distribution curves}

Fig. 15 is the curves of the frequency distribution functions of $V$ which are plotted from the original data above arranged and from the numerical evaluation by the Fourier analysis. In the same way Fig. 16 is the frequency distribution curves of $\phi$.

Comparing the curves from the original data with those from the Fourier analysis, we find that they do not coincide strictly. First that is because, as was mentioned above, 


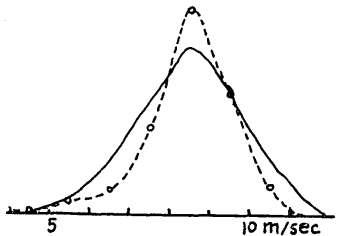

Fig. 15. Frequency distribution curves of wind speed. Full line : theoretical one Dashed line: observed one.

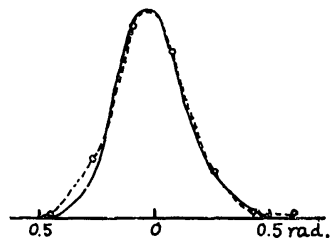

Fig. 16. Frequency distribution curves of wind direction. Full line: theoretical one Dashed line: observed one. the turbulent field is not always perfectly homogeneous and isotropic, and therefore the assumptions of homogeneity and isotropy which are adopted in the main theoretical investigation are not necessarily adequate to the actual phenomena. Secondly because of arrangement of the original data with which, in this case, we divided the total period into six parts. If we adopted another method of arrangement of the original data, we would slightly different curves of the frequency distribution functions. Thirdly because of small numbers of the used data, the curves are not always precise especially in both outer regions of the curves.

The discrepancies between the curves from the original data and those from the Fourier analysis, however, are not essential. Roughly speaking, they seem to obey the results of the present theory.

\section{The comment to diffusivity}

In the actual atmosphere the diffusivity in the vertical has been studied by many authors, but that in the horizontal is not studied so well as in the vertical. Here we shall give a technique to determine a horizontal diffusivity using the observation of the wind at one station. On the assumption that the turbulence is stationary, homogeneous and isotropic, the eddy diffusivity $\varepsilon$ is given

$$
\varepsilon=\overline{u^{2}} \int_{0}^{t} R_{L}(\xi) d \xi,
$$

where $R_{L}(\xi)$ is a Lagrangian correlation function. The discussion in detail of $R_{L}(\xi)$ is not our main purpose. The general nature of the integral is

$$
\int_{0}^{t} R_{L}(\xi) d \xi= \begin{cases}t-g(t) & t \leq \tau_{0} \\ k & t>\tau_{0} .\end{cases}
$$

The constant $k$ depends on $\tau_{0}$, where $\tau_{0}$ is the time length when $R_{L}(\xi)$ vanishes significantly. The mean square velocity is shown

$$
\begin{aligned}
\overline{u^{2}}= & \int_{0}^{U_{m}} U^{2} \hat{F}_{n}(U) U d U d \theta \\
= & 2 \sigma^{2}\left(1+4 \alpha \sigma^{2}\right)-\left(U_{m}{ }^{2}+2 \sigma^{2}+8 \alpha \sigma^{4}+4 \alpha \sigma^{2} U_{m}{ }^{2}\right. \\
& \left.+\alpha U_{m}{ }^{4}\right) \exp \left[-\frac{U_{m}^{2}}{2 \sigma^{2}}\right] .
\end{aligned}
$$

If we use the Lagrangian correlation functions which were derived by Taylor (1921), Sutton (1932) and Inoue (1950, 1951, 1952)Ogura (1952), $g(t)$ become as follows respectively:

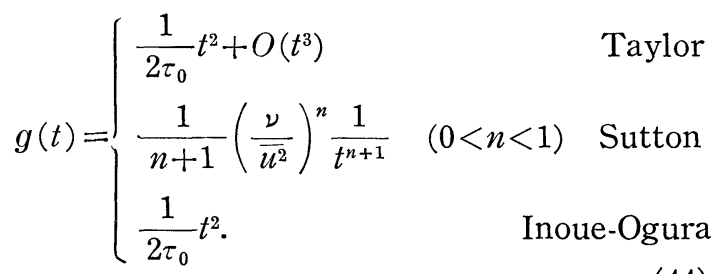

Taylor's correlation function and InoueOgura's one coincide in their first terms of $g(t)$. In Sutton's correlation function, $n$ changes according to the meteorological conditions such as instability and so on.

In our observed data analysis, $\overline{u^{2}}$ becomes approximately from (43),

$$
\overline{u^{2}} \simeq 2 \sigma^{2}=2.56 \mathrm{~m}^{2} / \mathrm{sec}^{2}=2.56 \times 10^{4} \text { c. g. s. } .
$$

Using Inoue-Ogura's correlation function, $\varepsilon$ becomes

$$
\varepsilon= \begin{cases}2.56 \times 10^{4} \times\left(t-\frac{t^{2}}{2 \tau_{0}}\right) & t \leq \tau_{0} \\ 2.56 \times 10^{4} \times \frac{\tau_{0}}{2} & t>\tau_{0} .\end{cases}
$$

Table 2. Eddy diffusivity (c.g.s. unit)

\begin{tabular}{cc|c|c}
\hline$t(\mathrm{sec})$ & $\tau_{0}(\mathrm{sec})$ & 10 & 100 \\
\hline 1 & $2.56 \times 10^{4}$ & $2.56 \times 10^{4}$ \\
\hline 10 & $1.28 \times 10^{5}$ & $2.43 \times 10^{5}$ \\
\hline 50 & $1.28 \times 10^{5}$ & $9.60 \times 10^{5}$ \\
\hline 100 & $1.28 \times 10^{5}$ & $1.28 \times 10^{6}$ \\
\hline
\end{tabular}


Table 2 are the eddy diffusivity which are derived from above equation (46).

It is important to determine a Lagrangian correlation function more decisively. This study will be performed in near future.

\section{References}

Ertel, H., 1929: Die Richtungsschwankung der horizontalen Windkomponente im turbulenten Luftstrom. Gerl. Beitr. Geophys. 23, 15-21.

Hesselberg, T. and Björkdal, E., 1929: Über das Verteilungsgesetz der Windunruhe. Beitr. Phys. fr. Atmos, 15, 121.

Inoue. E., 1950: The turbulent diffusion in the atmosphere (1), J. meteor. Soc. Japan, 28, 441441. atmosphere (11), J. meteor. Soc. Japan, 29, 246-253.

, 1952: Some remarks on the vertical diffusion of smoke near the ground, $J$. meteor. Soc. Japan, 30, 216-222.
Ogura, Y., 1952a: The theory of turbulent diffusion in the atmosphere (I), J. meteor. Soc. Japan, 30, 23-28.

- $1952 \mathrm{~b}$ : The theory of turbulent diffusion in the atmosphere (II), J. meteor. Soc. Japan, 30, 53-58.

$-1952 \mathrm{c}$ : The theory of turbulent diffusion in the atmosphere (III), J. meteor. Soc. Japan, 30, 386-397.

Priestley, C.H.B., 1959a: Turbulent transfer in the lower atmosphere. Univ. of Chicago Press. 130 pp.

-, 1959b; The isotropic limit and the microscale of turbulence. Advances in geophysics, 6, 97-100.

Sutton. O.G., 1932: A theory of eddy diffusion on the atmosphere, Proc. Roy. Soc., 135, 143-165.

Syōno, S., 1953: Frequency distribution functions of velocities in turbulent flow. J. meteor. Soc. Japan, 31, 299-305.

Taylor, G.I., 1921: Diffusion by continuous movements, Proc. London Math. Soc., Ser. 2, 196212 .

\title{
乱流における速度と方向の頻度分布について
}

\author{
正野重方・田中 浩 \\ 東京大学理学部地球物理学教空
}

二次元乱流飞招いて，それを等方的，一様，定常的と仮定した場合，平均流を屯含めた風速頻度分布，風向頻度分 布を“迷い歩き”の理論を沁用して求めた。この場合風速のフーリエ級数䦘開と怙ける位相任がランダムであり, フーリエ成分は相互と独立であると仮定している。

さら飞水平方向飞扔ける拨散係数に関して多少の考察を行ない, 最後飞束海村の日本原子力研究所の気象塔で得ら れた風速，風向の資料を解析し，特に風向に関しては理諭とよく一致することを示した。 